\title{
FACE RECOGNITION UNDER VARYING BLUR IN AN UNCONSTRAINED ENVIRONMENT
}

\author{
Anubha Pearline.S $\mathbf{1}$, Hemalatha. $\mathbf{M}^{\mathbf{2}}$ \\ ${ }^{1}$ M.Tech, Information Technology,Madras Institute of Technology, TamilNadu,India, \\ anubhapearl@gmail.com \\ ${ }^{2}$ Assistant Professor, Information Technology,Madras Institute of Technology, TamilNadu,India, email:, \\ hemalatha.ch@gmail.com
}

\begin{abstract}
Face recognition (FR) system is one of the prominent research areas in pattern recognition and computer vision because of its major challenges. Few challenges in recognizing faces are blur, illumination, and varied expressions. Blur can be uniform and non-uniform. Most probably, blur occurs while taking photographs using cameras, mobile phones. Non-uniform blur happens in images taken using any handheld image devices. Recognizing or handling a blurred image in a face recognition system is generally hard. Most algorithms like blind deconvolution, deblurring, etc. are present and their problems have been discussed. To solve the issue of blur, Blur Robust Face recognition algorithm (BRFR) has been done. In Blur Robust Face Recognition, the Local Binary Pattern (LBP) features were extracted from the blurred test image. For each image in the set of training images, the value for optimal Transformation Spread Function (TSF) was found in order to form a transformed image. The LBP features were extracted from the transformed image. Both the LBP features of blurred test image and transformed training image were compared to find the closest match. Yale face database and JAFFE database were used for evaluation. By varying the Blur levels for the test image, the results for both the datasets were obtained.
\end{abstract}

Keywords: Face Recognition, Blur, Transformation Spread Function, Local Binary Pattern.

\section{INTRODUCTION}

Image processing is processing of images using certain operations for which the input and output are images.In image processing, Face Recognition (FR) is one of the eminent research areas in pattern recognition and computer vision due to its numerous applications in the area of biometrics, information security, access control and surveillance system.

FR system is used in our day to day life such as in Facebook, mobile applications, login software. FR system identifies and authenticates people through face images. Other applications of FR are,

1. Biometric Identification and Authentication eg. voter identity cards, aadhar cards, passport identity and pan cards.

2. FR is used for security purposes in airports, seaports, ATM (Automated Teller Machines) machines.

3. Surveillance cameras

4. FR system is used in image database investigations such as finding missing criminals, children, immigrants and licensed drivers by checking the image databases.

5. FR systems can be used to analyze scenes from videos by looking out for any odd occurrence of specific vehicles and specific faces.

Gender classification, age classification, recognizing facial expressions, recognizing facial feature and tracking are some of the related applications of FR. Facial feature recognition and detection are used for tracking and monitoring a vehicle driver's eyes and his fatigue. Facial expression recognition can be used for monitoring patients in intensive care units. FR is used with biometrics like iris, fingerprint, ear, speech and gait recognition. Basic steps in Face Recognition are given in the following chart in Fig.1. Any input image is detected for which features are extracted and features are classified using any suitable classification technique. In FR, as faces are dealt it is important to identify images if they are faces or not and this is known as face detection. But, face detection step is not a compulsory task to be done, because faces are present in training images in some form or the other[1].

Facial features are extracted using various features like Gabor features, HoG (Histogram of Gradients), DoG (Difference of Gradients). Recognizing faces is the final step in FR.

There are two types of application in FR. One is identification and the other is verification. For identification problem in FR, from a database of known persons, the input face is being compared to find the identity of the image. In FR, the claimed identity of the input face is either confirmed or rejected in verification[2]. 


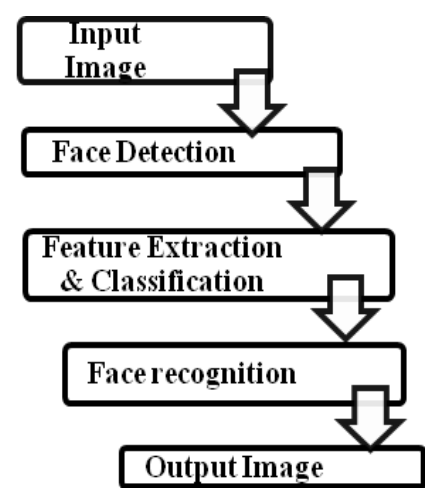

Fig-1 FR Step

An FR system should automatically detect a face from an image. However, the accuracy of FR systems worsens quite rapidly in unconstrained settings such as illumination, expression and pose variations. This involves extracting features and then recognizing it, irrespective of illumination, expression, ageing, transformations (translation, rotation and scaling of the image) and pose variations, which is a difficult task.

\subsection{Blur}

Blur is common while taking photographs using cameras and mobile phones. Blur can be uniform and non-uniform. Normally Non-uniform Blur occurs in images taken using any handheld image devices. Recognizing or handling a blurred image in a face recognition system is generally hard [3].

\subsection{Illumination}

Photographs or images occur with illumination and varies as good and dark illumination. Under different lighting conditions, there are more chances for face recognition system to identify person wrongly. Illumination may be as left light, center light and right light.

\subsection{Expression}

Expressions are a form of non-verbal communication [4]. There are various facial expressions such as happiness, sad, surprise, fear, anger which changes or deforms the faces from normal images. Recognizing faces with facial expressions is an exciting task.

\subsection{Pose Variations}

FR of frontal face images is easier than images with pose variations. Small pose variations in angles can be easily handled than larger variations.

\section{LITERARY WORKS}

Soodeh Nikhan et al.[5] proposed a system for local illumination insensitive face identification algorithm which is the combination of LBP and LPQ (Local Phase Quantisation). LBP and LPQ are two illumination insensitive local descriptors. This method was found to be easy to execute since it does not require training and image reconstruction. But, the method was observed to be computationally cost effective.

P.Vageeswaran et al. [6] considered a blur and illumination robust algorithm by showing that the set of all images formed from a face image by blurring it and on changing the illumination conditions forms a bi-convex set. Also, amalgamating a particular learning approach like SVM into this formulation would be a very promising direction for future work.

R. Gopalan et al.[7] showed that using Grassmann manifold, a subspace was formed.The subspace obtained was a result of convolutions of an image. This convolution contained a complete set of orthonormal basis functions representing the blur kernels. These blur kernels were invariant to blur under some assumptions. Robust face recognition would be an interesting topic under light and pose variations along with various blur values.

V. M. Patel et al.[8] proffered an FR algorithm dependent on dictionary learning methods. These methods were robust to changes in lighting and pose. Future work is to develop and analyze the accuracy of a discriminative dictionary learning algorithm which is robust to pose, expression and illumination variations. Albedo estimation mechanisms are sensitive to facial expressions. Discriminative dictionary increases the overall computational complexity making the real-time process handling very difficult. Discriminative dictionaries are sensitive to noise.

Yu-Wing Tai et al.[9] has modeled the blurred image as an integration of the clear scene under a sequence of planar projective transformations i.e., homography that describes the camera's path. A fundamental limitation to their algorithm is that the high-frequency details which have been lost during the motion blur process cannot be recovered. The algorithm can only recover the hidden details which remains inside the motion blur images. Also, it has not dealt with moving or deformable objects or scenes with occlusion or disocclusion, or with significant depth variation.

A technique for FR in varied pose was schemed by Changxing Ding et al.[10]. For this, they have used Patchbased Partial Representation (PBPR) and Multi-Task Feature Transformation Learning (MtFTL) together. It can handle pose variations upto 60 degrees. Changxing Ding did not consider blur for face recognition. Also, this technique efficiently utilizes unoccluded face textures.

\section{SYSTEM DESIGN}

\subsection{Problem Definition}

Given a set of training images, $g_{c}$ and a test image $p$, the identity of $p$ has to be found. Test image, 'p' may be Blurred, illuminated, along with varied expressions. The matrix $A_{c}$ for each training face was generated. Test image, $p$ can be summarized as the convex combination of the columns of one of these matrices. For recognition task, the optimal TSF $\left[\mathrm{T}_{\mathrm{c}}\right]$ for training images is computed. 
The following architecture in Fig. 2 is for BRFR algorithm which is Blur invariant face recognition. Here, for the blurred test image LBP features were extracted. For a set of training image, optimal Transformation Spread Function (TSF) was used to form transformed image. LBP features was extracted from the transformed image. Both the features were compared to find the identity of the image.

\subsection{Blur Invariant Face Recognition}

If there were $1,2 \ldots \mathrm{C}$ face classes, then each class $\mathrm{c}$ contains $\mathrm{g}_{\mathrm{c}}$, which denotes training images of that class. Also, the blurred test image, $\mathrm{p}$ belongs to any of the $\mathrm{C}$ classes. For each training face, transformed images have been formed and these images forms the column of the matrix $A_{c}$. The test image's identity is obtained using reconstruction error $r_{c}$ in (1). The identity of $p$ is found with the one having minimum $r_{c}[3]$.

$$
\mathrm{r}_{\mathrm{c}}=\min _{\mathrm{T}}\left\|\mathrm{p}-\mathrm{A}_{\mathrm{c}} \mathrm{T}\right\|_{2}^{2}+\beta\|\mathrm{T}\|_{1} \text {, subject to } \mathrm{T} \geq 0
$$

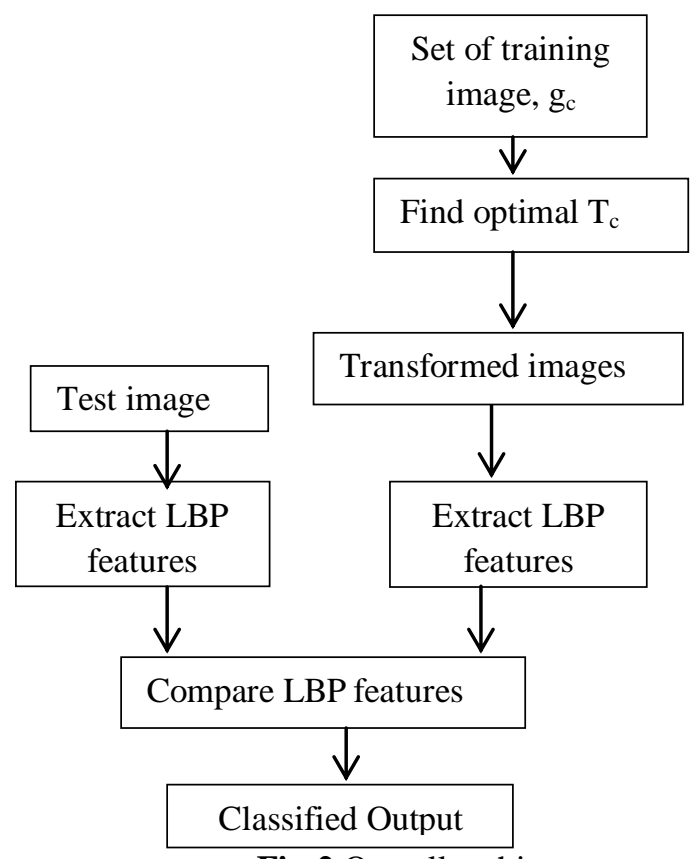

Fig-2 Overall architecture

Even though all the pixels contain equal weights, every region in the face does not convey equal amount of information. For this, a weighting matrix, $\mathrm{W}$ was introduced. This value was introduced to make algorithms robust to variations like misalignment. For training the weights, training and test image folders of the dataset were bused. The test image folder was Blurred with Gaussian kernel, $\sigma=4$. The images taken were partitioned as block patches using DRBF through which recognition rates were obtained independently. The LBP features were used in DRBF. LBP features of both blurred test images and training images have been considered for the recognition rate. The regions around the eyes are given the highest weights [6].In Fig.3, the white region has highest weights, the other colours have lower weights and the darkest regions have very low weights.
The reconstruction error, $r_{c}$ becomes as follows when the weighting matrix has been used,

$r_{c}=\min T\left\|W\left(g-A_{c} T\right)\right\|_{2}^{2}+\beta\|T\|_{1}$, subject to $T \geq 0$

The reconstruction error, $r_{c}$ has been sensitive to small pixel misalignments and hence, it is not preferable.Test image, $p$ represents the input for which LBP features were extracted.

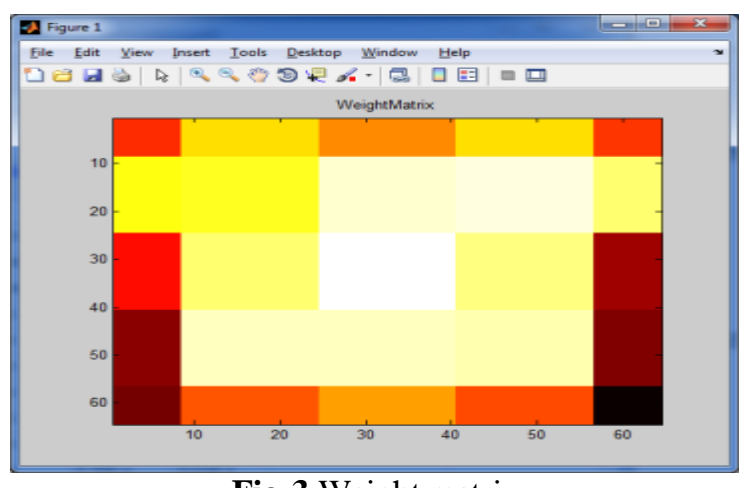

Fig-3 Weight matrix

For every train image, $\mathrm{g}_{\mathrm{c}}$, Blur was created through optimal Transformation Spread Function(TSF) $\mathrm{T}_{\mathrm{c}}$. This optimal TSF was found by,

$\mathrm{T}_{\mathrm{c}}=\arg \min _{\mathrm{T}}\left\|\mathrm{W}\left(\mathrm{p}-\mathrm{A}_{\mathrm{c}} \mathrm{T}\right)\right\|_{2}^{2}+\beta\|\mathrm{T}\|_{1}$, for $\mathrm{T} \geq 0$

Here,

$\mathrm{T}_{\mathrm{c}}$, optimal TSF

$\mathrm{W}$, weighting matrix

$\mathrm{p}$, represents Blurred test image

$A_{c}$, matrix for each training face

$\mathrm{T}$, vector of weights

$\mathrm{W}$, a weighting matrix uses weights similar to LBP. The difference between $g$ and $\mathrm{A}_{\mathrm{c}} \mathrm{T}$ were calculated. Vector of weights denoted by $\mathrm{T}, \mathrm{T} \geq 0$ is always rounded off to nearest integer $1\left(\|T\|_{1}\right)$. ' $A$ ' represents matrix, $A$ is a part of $R($ Real Numbers) belonging to $\mathrm{N}^{*} \mathrm{~N}_{\mathrm{T}}$, which was formed by total number of transformations and overall number of pixels in the image, $\mathrm{N}_{\mathrm{T}}$ and $\mathrm{N}$. For each training face, a matrix $\mathrm{A}_{c}$, was generated. Finally, optimal TSF, $\mathrm{T}_{\mathrm{c}}$ has been identified using equation (3).

Training images and blurred test images were partitioned into non-overlapping rectangular regions for which LBP histograms were extracted and histograms were concatenated for building a global descriptor. LBP features of both training image and blurred test image are being compared using to find if they almost match with each other. The output is the identity of the test image. The following column contains algorithm for BRFR.

\begin{tabular}{|c|c|}
\hline \multicolumn{2}{|c|}{ ALGORITHM: BLUR ROBUST FACE RECOGNITION } \\
\hline & $\begin{array}{l}\text { Гest image } \mathrm{p} \text { and a set of training images } \mathrm{g}_{\mathrm{c}} \text {, } \\
\text {.. C. }\end{array}$ \\
\hline & Identity of test image, $\mathrm{p}$. \\
\hline 1. & For each $g_{c}$, \\
\hline 2. & Find optimal TSF $\mathrm{T}_{\mathrm{c}}$ by solving equation (3). \\
\hline 3. & Blur every $g_{c}$ with its corresponding $T_{c}$. \\
\hline 4. & Extract LBP features of blurred $g_{c}$. \\
\hline & Compare LBP features of $\mathrm{p}$ and transformed $\mathrm{g}_{\mathrm{c}}$. \\
\hline & Find closest match of $\mathrm{p}$. \\
\hline
\end{tabular}




\subsection{Local Binary Pattern (LBP)}

LBP is a simple, productive texture operator. The LBP texture operator is an outstanding path in a variety of applications because of its discriminating power and computational simplicity. LBP's property, computational simplicity had made it possible for analyzing images in challenging real-time settings. In traditional divergent statistical and structural models for texture analysis, LBP can be seen as a unifying approach.

LBP has been used in BRFR system as it is insensitive to blur, misalignment, illumination and varied poses. LBP is used for FR, image retrieval, biomedical image analysis and texture analysis. Many applications using LBP are biometrics, including eye localization, iris recognition, fingerprint recognition, palm print recognition, gait recognition, facial age classification, and facial expression recognition.

Neighbourhoods of different sizes were extended to be used in LBP operator. Circular neighbourhood and bilinear interpolating values being used at non-integer pixel coordinates allows any radius and number of pixels in the neighbourhood. A complementary contrast measure in the local neighbourhood is the gray scale variance. In the following eqn. (4), the notation ( $\mathrm{P}, \mathrm{R})$ has been used for pixel a neighbourhood $\mathrm{P}$ is represented as sampling points on a circle of radius of $R$.

$$
\mathrm{LBP}_{\mathrm{P}, \mathrm{R}}=\sum_{\mathrm{p}=0}{ }^{\mathrm{P}-1} \mathrm{~s}\left(\mathrm{~g}_{\mathrm{p}}-\mathrm{g}_{\mathrm{c}}\right) 2 \mathrm{p}, s(t)=\left\{\begin{array}{l}
1, t \geq 0 \\
0, o \cdot \mathrm{W}
\end{array}\right.
$$

Here,

$\mathrm{g}_{\mathrm{c}}$, denotes gray value of centre pixel in the local neighbourhood.

$\mathrm{g}_{\mathrm{p}}, \mathrm{p}=0,1, \ldots, \mathrm{P}-1$, represents the gray values of neighbourhood pixels.

Using the above equation (4), the binary codes have been computed. Fig-4 shows LBP of a facial image[11].

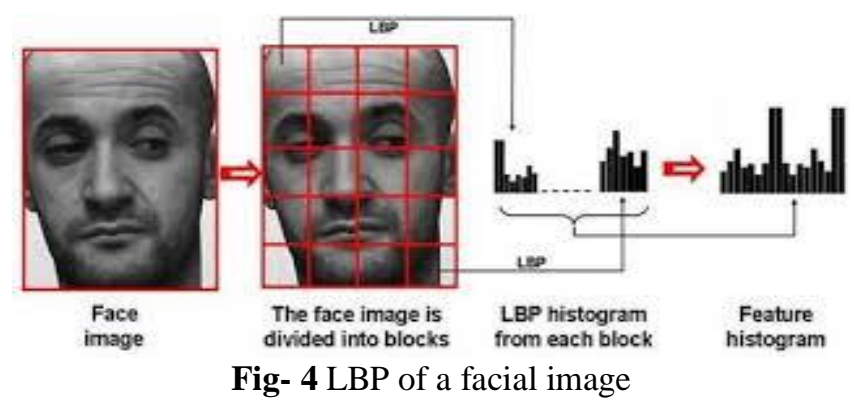

\subsection{K-Nearest Neighbour Classifier}

$\mathrm{K}$-nearest neighbour (KNN) is one of the elementary machine learning techniques. KNN is a type of instancebased learning or lazy learning as its function is approximated locally and delayed until classification.KNN has been found to be used in applications such as text mining, text categorization, finance, medicine and agricultural related fields. It requires no previous awareness about the distribution of data.This rule simply retains the entire training set during learning and assigns to each query a class represented by the majority label of its k-nearest neighbours in the training set.The simplest form of KNN when $\mathrm{k}=1$ is Nearest Neighbour rule (NN). In this method, each sample should be classified similarly to its surrounding samples. Hence, if the classification of a sample is unknown, then it could be figured out by considering the classification of its nearest neighbour samples. For a given unknown sample and a training set, all the distances between the unknown sample and all the samples in the training set were computed. The distance with the smallest value corresponds to the sample in the training set closest to the unknown sample. Therefore, the unknown sample may be classified based on the classification of this nearest neighbour. One of the very famous distances metric is Euclidean distance. Euclidean distance can be represented as,

$$
\mathrm{D}(\mathrm{a}, \mathrm{b})=\sum_{i=1}^{n} \sqrt{(b i-a i)}
$$

Where,

$\mathrm{D}(\mathrm{a}, \mathrm{b})$, denotes distance between $\mathrm{a}$ and $\mathrm{b}$,

$\mathrm{a}_{\mathrm{i}}$ and $\mathrm{b}_{\mathrm{i}}$, represents two points, $1<\mathrm{i} \leq \mathrm{n}$

Generally, the other distance measures used for classifying are Euclidean squared, Chebychev, city block. [12]

Here in the Fig.5, the test sample i.e. green circle should be classified either to the first class of blue squares or to the second class of red triangles. If $k=3$ i.e. within the solid line circle, it is assigned to the second class because there are 2 triangles and only 1 square inside the inner circle. If $\mathrm{k}=5$ i.e. within dashed line circle, it is assigned to the first class as there has been 3 squares and 2 triangles in the outer circle.

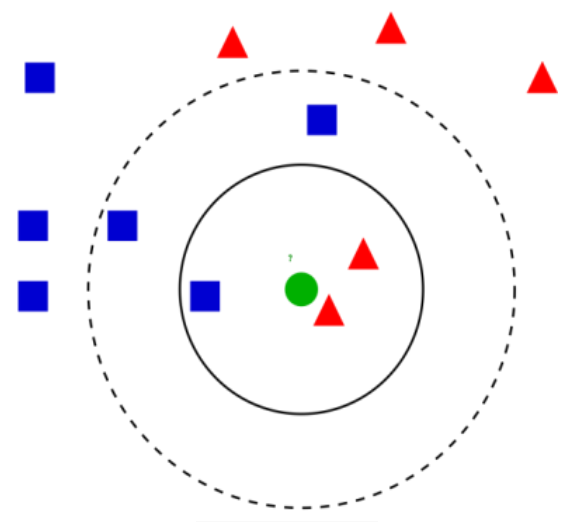

Fig- 5: KNN

\section{IMPLEMENTATION AND PERFORMANCE} ANALYSIS

\subsection{Datasets}

For experiments, two datasets have been used. One is Yale face database [13] and the other is JAFFE database [14]. The datasets have been segregated as training set and testing set. The trained images, forms the training set. The test images, forms the testing set. The images were separated into subjects' folders in the training set and test image set. 


\subsection{Performance Analysis}

The FR system was checked in two conditions, i.e. Gaussian and motion blur. Gaussian blur has been used for uniform blur. Motion blur has been used for non-uniform blur.

The following graph Chart-1 shows the recognition results from a Yale face database [13] and JAFFE database [14] using Gaussian blur. The sigma values were varied for test images as 15, 20, and 30. From the Yale face database, the recognition rate obtained was $66.66 \%$. The results are because of illumination effects in the dataset. For JAFFE dataset, the results obtained were $100 \%$ for each sigma value such as 15,20 , and 30 .

By varying values of len pixels 15, 20, 30 using motion blur, the following results were obtained. When len pixels were $15,20,30$ the recognition rate observed in the Yale face database was $66.66 \%$. For JAFFE dataset, the recognition rate was observed as $100 \%$ for all the len pixels 15, 20, and 30. The following graph Chart-2 shows the recognition results for both the datasets.

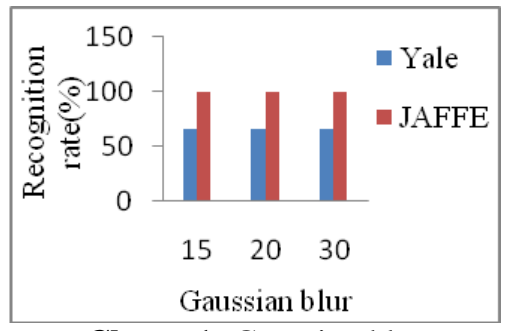

Chart- 1: Gaussian blur

Recognition rates for both blurs, Gaussian blur and motion blur were similar for the two datasets. In JAFFE dataset, each image taken had a similar type of illumination. While in Yale face dataset for some of the images, illumination varied. When the blur levels were increased further, the results decrease.

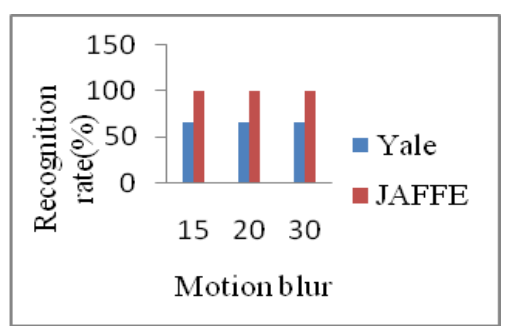

Chart-2: Motion blur

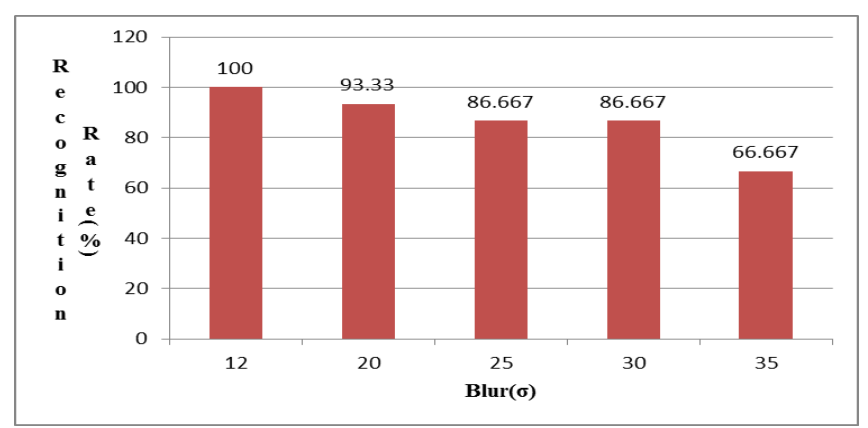

Chart- 3: Results of BRFR using Weight,W
The above Chart-3 shows the results of BRFR using weighting matrix, $\mathrm{W}$ for yale face database. Here, blur levels are increased in the range as 12, 20, 25, 25, 30, 35. These values are of sigma parameter.

The below figure, Chart-4 shows the results of BRFR without using weighting matrix, W. Blur levels are increased in the range as $12,20,25,25,30,35$.

These values are of sigma parameter. In Chart- 3 and Chart4 , $\mathrm{x}$-axis holds blur values whereas, $\mathrm{y}$-axis holds recognition rate. When sigma values of images are increased, the recognition rates recorded are $100 \%, 93.33 \%, 86.667 \%$, $86.667 \%$, 66.667\% in both charts, Chart- 3 and Chart4.Results for both these figures were taken from yale face database.

Chart- 4: Results of BRFR without W

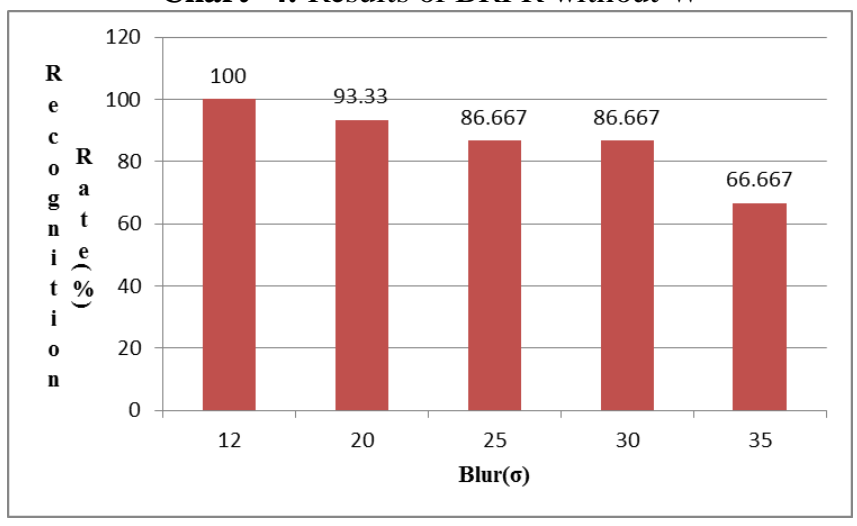

\section{CONCLUSION}

A FR system which is robust to blur was developed using BRFR algorithm. In this algorithm, the LBP features were extracted from the blurred test image. Each and every image in the training set was transformed using optimal TSF and their LBP features were extracted. LBP features of images were compared to find the best match. BRFR algorithm was implemented and their results were discussed in section 4 for the two datasets Yale face database and JAFFE. BRFR gives good results when the probe and training set have images of the same illumination. The system works efficiently and is robust to conditions like blur. The results will be improved when illumination and expression from faces are removed.

\section{FUTURE WORK}

Even though the issue of blur was not completely solved using BRFR, few problems such as illumination, expression variations still remains as a hindrance to FR system. As future work, this FR system can be extended to other challenges like occlusion, illumination, and expressions.

\section{REFERENCES}

[1]. W. Zhao, R. Chellappa, P. J. Phillips, and A. Rosenfeld, "Face recognition: A literature survey", ACM Computing Survey, vol. 35, no. 4, pp. 399-458, Dec. 2003.

[2]. Miss.A.D.Kandelkar, Prof.P.A.Tija, "Survey on blur 
Face Recognition Techniques", International Journal For Research In Emerging Science And Technology, Volume-2, Special Issue-1, pp. 606-609, Mar. 2015.

[3]. Abhijith Punnappurath and Ambasamudram Narayanan Rajagopalan,"Face Recognition Across Non-Uniform Motion Blur, Illumination, and Pose", IEEE Transactions On Image Processing, Vol. 24, No. 7, pp. 2067-2082, Jul. 2015.

[4]. Claude C. Chibelushi, Fabrice Bourel, "Facial Expression Recognition: A Brief Tutorial Overview",school of Computing, Staffordshire University, 2002.

[5]. Soodeh Nikhan and Majdi Ahmadi, "Local gradientbased illumination invariant face recognition using local phase quantisation and multi-resolution local binary pattern fusion", IET, Image Processing, Volume: 9, Issue: 1,pp. 12 - 21, Jun. 2015.

[6]. P.Vageeswaran, K. Mitra, and R. Chellappa, "Blur and illumination robust face recognition via set-theoretic characterization", IEEE Transactions On Image Processing, vol. 22, no. 4, pp. 1362-1372, Apr. 2013.

[7]. R. Gopalan, S. Taheri, P. Turaga, and R. Chellappa, "A blur-robust descriptor with applications to face recognition," IEEE Trans. Pattern Anal. Mach. Intell., vol. 34, no. 6, pp. 1220-1226, Jun. 2012.

[8]. M. Patel, T. Wu, S. Biswas, P. J. Phillips, and R. Chellappa, "Dictionary-based face recognition under variable lighting and pose", IEEE Trans. Inf. Forensics Security, vol. 7, no. 3, pp. 954-965, Jun. 2012.

[9]. Y.W. Tai, P. Tan, and M. S. Brown, "Richardson-Lucy deblurring for scenes under a projective motion path", IEEE Trans. Pattern Anal. Mach. Intell., vol. 33, no. 8, pp. 1603-1618, Aug. 2011.

[10]. Changxing Ding, Chang Xu, and Dacheng Tao, "Multitask Pose-Invariant Face Recognition", IEEE Transactions on image processing, vol. 24, no. 3, Mar. 2015.

[11].T. Ahonen, A. Hadid, and M. Pietikainen, "Face description with local binary patterns: Application to face recognition", IEEE Transactions on Pattern Analysis and Machine Intelligence, vol. 28, no. 12, pp. 2037-2041, Dec.2006.

[12].Sadegh Bafandeh Imandoust And Mohammad Bolandraftar," Application of K-Nearest Neighbor (KNN) Approach for Predicting Economic Events: Theoretical Background", Int. Journal of Engineering Research and Applications, Vol. 3, Issue 5, pp.605-610, Sep-Oct 2013.

[13].P. N. Bellhumer, J. Hespanha, and D. Kriegman, "Eigenfaces vs. fisherfaces: Recognition using class specific linear projection", IEEE Transactions on Pattern Analysis and Machine Intelligence, Special Issue on Face Recognition, vol. 19,no. 19, pp.711720, Jul. 1997.

[14].Michael J. Lyons, Julien Budynek, \& Shigeru Akamatsu, "Coding Facial Expressions with Gabor Wavelets", 3rd IEEE International Conference on Automatic Face and Gesture Recognition, pp. 200-205, Apr.,1998.

\section{BIOGRAPHIES}

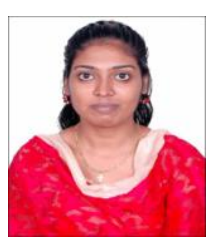

Ms.S.Anubha Pearline, received B.E. in Computer science and Engineering, in 2015. She is currently pursuing M.Tech, at Anna University, Madras Institute of Technology Campus. Her area of interest lies in the area of Image Processing.

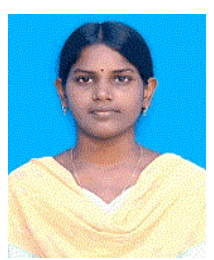

Ms.M.Hemalatha, M.E. [Communication \& Networking] is an Assistant Professor at Anna University, Madras Institute of Technology Campus. She did her M.E at Madras Institute of Technology and B.Tech [Information Technology], at Panimalar Engineering College. Her area of interest is on Image Processing and Networking. 